\title{
WRN Cys1367Arg polymorphism is not associated with skull base chordoma
}

\author{
KE WANG $^{1 *}$, LIANG WANG $^{1^{*}}$, JIE FENG $^{2}$, SHUYU HAO $^{1}$, KAIBING TIAN $^{1}$, ZHEN WU $^{1}$, \\ LIWEI ZHANG $^{1}$, GUIJUN JIA ${ }^{1}$, HONG WAN ${ }^{2}$ and JUNTING ZHANG ${ }^{1}$ \\ ${ }^{1}$ Skull Base and Brainstem Tumor Division, Department of Neurosurgery, Beijing Tian Tan Hospital; \\ ${ }^{2}$ Beijing Neurosurgery Institute, Capital Medical University, Beijing 100050, P.R. China
}

Received January 24, 2014; Accepted March 4, 2014

DOI: $10.3892 /$ br. 2014.275

\begin{abstract}
Skull base chordoma is a rare tumor with unknown risk factors. Werner syndrome, which is caused by a mutation in the WRN gene, is a disease of progeria, resembling the pathological process of aging. The present study aimed to provide data on the possible association between skull base chordoma and the single-nucleotide polymorphism (SNP) rs1346044 of the WRN gene. Between July, 2010 and September, 2012, a total of 65 patients with pathologically confirmed skull base chordoma and 65 control subjects were enrolled in this case-control study. The clinical data of the skull base chordoma patients were documented and the rs1346044 site in all the enrolled subjects was analyzed by sequencing and statistically compared using SPSS software. The A allele was the dominant allele of the rs1346044. The comparisons of genotype distributions and allele frequencies did not reveal any significant difference between the groups $[\mathrm{P}=0.383,95 \%$ confidence interval (CI): 0.346-1.505]. The clinicopathological factors were assessed and no statistically significant difference was observed. In conclusion, the present study suggested that there is no association between rs1346044 SNP and skull base chordomas, at least in the population analyzed.
\end{abstract}

\section{Introduction}

Chordoma is a rare neoplasm believed to be derived from the remnants of the notochord. Chordoma is a low-to-middle malignancy tumor, mainly seen along the spinal axis, with a prevalence of $0.08 / 100,000$ individuals. Skull base chordoma represents $\sim 1 / 3$ of chordoma cases (1). The peak incidence of

Correspondence to: Dr Junting Zhang, Skull Base and Brainstem Tumor Division, Department of Neurosurgery, Beijing Tian Tan Hospital, Capital Medical University, 6 Tiantan Xili Road, Beijing 100050, P.R. China

E-mail: zhangjunting2003@aliyun.com

*Contributed equally

Key words: WRN gene, skull base chordoma, single-nucleotide polymorphism, rs1346044 skull base chordomas is mainly reported in the 3rd and 4th decades of life, with a slight predominance in men (2,3). Skull base chordoma has a poor prognosis, with a median survival of 6.29 years and the 5-, 10- and 20-year survival decreasing precipitously to $67.6,29.9$ and $13.1 \%$, respectively, regardless of ethnicity and gender $(1,2)$.

Werner syndrome, caused by a mutation in the WRN gene, is an autosomal recessive disease characterized by the early onset of normal aging, including wrinkling of the skin, graying of hair, cataract, diabetes, osteoporosis and development of tumors, such as meningioma (4). The WRN protein is a member of the RecQ family of helicases, which is crucial for the maintenance of telomere structure and the initiation of DNA damage response following telomere disruption. Mutations in the WRN gene lead to a loss of function of the WRN protein and a breakdown in genome integrity (5). Investigation of WRN, including mutations, deletions and polymorphisms, are performed in a number of tumors and age-related diseases (5). The WRN codon 1367, located in the short arm of chromosome 8, is a single-nucleotide polymorphism (SNP) site (rs1346044), where the allele A-to-G change leads to a cysteine-to-arginine substitution in the WRN protein $(5,6)$. Studies on the WRN Cys1367Arg reported an association with several diseases, such as cataract (6) and bone and soft tissue sarcomas (7); however, to the best of our knowledge, there has been no such investigation for chordoma.

The present study aimed to investigate the possible association between the Cys1367Arg (rs1346044) polymorphism in the WRN gene and skull base chordomas in the Han Chinese population.

\section{Materials and methods}

Study population. Between July, 2010 and September, 2012, a total of 65 patients with pathologically confirmed skull base chordoma, treated at the University Hospital of the Tian Tan Hospital, Capital Medical University, Beijing, China, were enrolled in this population-based case-control study, which was approved by the Ethics Committee of the Capital Medical University. The male:female ratio was 1.17:1 (35:30) and the mean age \pm SD was 37.9 \pm 14.4 years (range, $8-69$ years). A total of 65 healthy individuals were enrolled as the control group, with a male:female ratio of $1.17: 1(35: 30)$ and a mean age \pm SD of $43.7 \pm 10.8$ years (range, $25-79$ years). Blood samples $(5 \mathrm{ml})$ 
were collected from all the patients and control subjects. All the participants provided written informed consent prior to enrollment.

DNA extraction and genotyping. DNA extraction was performed using Proteinase $\mathrm{K}$ and phenol-chloroform, according to the TIANamp Blood DNA kit (DP318), Tiangen Biotech Co., Ltd. (Beijing, China). Subsequently, the DNA samples were successfully prepared and stored at $4^{\circ} \mathrm{C}$. The primers were designed by Sangon Biotech Co., Ltd. (Shanghai, China). The sequence containing the polymorphic site was amplified by polymerase chain reaction using the designed primers: forward, 5'-CCTTCCACCTTCCTTTCTACAG-3' and reverse, 5'-TGATGCCTACTTCTTCCTTGC-3'. Genotyping was performed by Sangon Biotech Co., Ltd. by sequencing.

Statistical analysis. The independence of allele frequency (Hardy-Weinberg equilibrium) was ensured with the Chi-square test. The genotype distribution and the allele frequencies between patients and control subjects were compared using the Chi-square and Fisher's exact tests. The odds ratios and $95 \%$ confidence intervals (CI) were calculated with a square test via the SPSS statistical software (SPSS Inc., Chicago, IL, USA).

\section{Results}

Hardy-Weinberg equilibrium assessment. The rs1346044 SNP was successfully detected in all the subjects, except one in the patient group (64 skull base chordomas and 65 controls). The genotype and allele frequencies were assessed for Hardy-Weinberg equilibrium in different ethnicities $(\mathrm{P}>0.05)$ and the results are summarized in Table I.

Gender and age characteristics. As there was a limited number of controls, as well as possible selection bias, similar control subjects for the rs1346044 site in the Han Chinese population in a study by Jiang et al (6) were used as a reference. In that study, there were 244 controls and the male:female ratio was 115:129 (0.89:1), with a mean age \pm SD of 60.2 \pm 5.7 years (Tables I and II).

Genotype and allele frequency comparson between cases and controls. When comparing the allele frequencies, the chordoma patients and the controls of the study by Jiang et al (6) exhibited no positive correlation ( $\mathrm{P}=0.610$; Table III). The A allele was the dominant allele of the rs1346044 SNP. In all the subjects enrolled, no GG genotype was detected and the frequencies of the GA and AA genotypes in the case group were 29.7 and $70.3 \%$, respectively, whereas in the control group they were 36.9 and $63.1 \%$, respectively. There was no positive association between the two groups $(\mathrm{P}=0.383$; Table III).

Genotype and allele frequency comparison by gender and clinicopathological characteristics. As regards analysis by gender, the male patients exhibited a positive correlation to the A allele, with a significance level of 0.009 (95\% CI: 0.092-0.729); however, the female patients exhibited no such correlation (Table IV).

The clinicopathological characteristics are presented in Table V. Association analyses were performed for chordoma cases only, by dividing the patients into two groups according
Table I. Hardy-Weinberg equilibrium of rs1346044 in different ethnicities.

\begin{tabular}{lcccc}
\hline rs1346044 & $\mathrm{G}$ & $\mathrm{A}$ & $\chi^{2}$ & P-value \\
\hline $\mathrm{HCB}^{\mathrm{a}}$ & 0.070 & 0.930 & - & - \\
$\mathrm{CEU}^{\mathrm{a}}$ & 0.296 & 0.704 & - & - \\
YRI $^{\mathrm{a}}$ & 0.106 & 0.894 & - & - \\
Case group $_{\text {Control group }}^{\mathrm{b}}$ & 0.15 & 0.85 & 0.21 & $>0.05$ \\
Control group $^{\mathrm{c}}$ & 0.13 & 0.82 & 3.33 & $>0.05$ \\
\hline
\end{tabular}

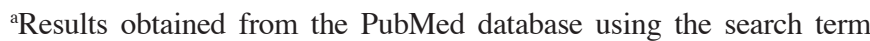
'SNP rs1346044'. ' Data from the present study. 'Data from the study by Jiang et al (6). HCB, Asian; CEU, European; YRI, Sub-Sahara African.

Table II. Gender and age characteristics of the case and control groups.

\begin{tabular}{lcccc}
\hline Groups & Total no. & Male & Female & $\begin{array}{c}\text { Age, years } \\
(\text { mean } \pm \text { SD) }\end{array}$ \\
\hline Case & $64^{\mathrm{a}}$ & 34 & 30 & $38.3 \pm 14.2$ \\
Control $^{\mathrm{b}}$ & 65 & 35 & 30 & $43.7 \pm 10.8$ \\
Control $^{\mathrm{b}}$ & 244 & 115 & 129 & $60.2 \pm 5.7$ \\
\hline
\end{tabular}

${ }^{a}$ rs 1346044 SNP was not detected in one patient. ${ }^{b}$ Data from the present study. ${ }^{C}$ Data from the control group from the study by Jiang et al (6).

Table III. Genotype and allele frequencies of WRNCys 1367Arg single-nucleotide polymorphism in the case group and two control groups for skull base chordomas.

A, Comparison with control group from the present study

\begin{tabular}{lcccc}
\hline Genotype & $\begin{array}{c}\text { Cases } \\
\mathrm{n}(\%)\end{array}$ & $\begin{array}{c}\text { Controls } \\
\mathrm{n}(\%)\end{array}$ & P-value & $95 \%$ CI \\
\hline G/G & 0 & 0 & 0.383 & $0.346-1.505$ \\
G/A & $19(29.7)$ & $24(36.9)$ & & \\
A/A & $45(70.3)$ & $41(63.1)$ & & \\
G 1367 Freq. & 0.15 & 0.18 & 0.436 & $0.398-1.487$
\end{tabular}

B, Comparison with control group from Jiang et al (6)

\begin{tabular}{lcccc}
\hline Genotype & $\begin{array}{c}\text { Cases } \\
\mathrm{n}(\%)\end{array}$ & $\begin{array}{c}\text { Controls } \\
\mathrm{n}(\%)^{\mathrm{a}}\end{array}$ & P-value & $95 \% \mathrm{CI}$ \\
\hline G/G & 0 & $5(2.1)$ & 0.254 & - \\
G/A & $19(29.7)$ & $54(22.1)$ & & \\
A/A & $45(70.3)$ & $185(75.8)$ & & \\
G 1367 Freq. & 0.15 & 0.13 & 0.610 & $0.498-1.506$ \\
\hline
\end{tabular}

aData from the control group from the study by Jiang et al (6). Freq., allele frequency; CI, confidence interval. 
Table IV. Genotype and allele frequencies of WRN Cys1367Arg single-nucleotide polymorphism by gender in the case and control groups for skull base chordomas.

\begin{tabular}{|c|c|c|c|c|c|}
\hline Gender & Genotype $^{\mathrm{a}}$ & Cases, n (\%) & Controls, n (\%) & P-value & $95 \% \mathrm{CI}$ \\
\hline \multirow[t]{3}{*}{ Male } & $\mathrm{G} / \mathrm{A}$ & $8(23.5)$ & $19(54.3)$ & 0.009 & $0.092-0.729$ \\
\hline & $\mathrm{A} / \mathrm{A}$ & $26(76.5)$ & $16(45.7)$ & & \\
\hline & G 177 Freq. & 0.12 & 0.32 & 0.006 & $0.115-0.719$ \\
\hline \multirow[t]{3}{*}{ Female } & $\mathrm{G} / \mathrm{A}$ & $11(36.7)$ & $5(16.7)$ & 0.080 & $0.860-9.745$ \\
\hline & $\mathrm{A} / \mathrm{A}$ & $19(63.3)$ & $25(83.3)$ & & \\
\hline & G 177 Freq. & 0.18 & 0.08 & 0.107 & $0.802-7.607$ \\
\hline
\end{tabular}

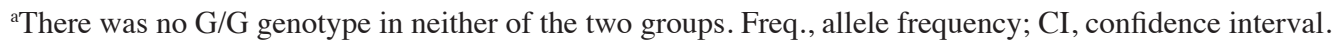

Table V. Association of rs1346044 genotype and clinicopathological characteristics in skull base chordoma.

\begin{tabular}{|c|c|c|c|c|c|c|c|c|}
\hline \multirow[b]{2}{*}{ Characteristics } & \multirow[b]{2}{*}{ Total } & \multicolumn{2}{|c|}{ Genotype $^{\mathrm{a}}$} & \multicolumn{2}{|c|}{ Allele count } & \multicolumn{2}{|c|}{ Frequency } & \multirow[b]{2}{*}{ P-value } \\
\hline & & G/A & $\mathrm{A} / \mathrm{A}$ & $\mathrm{G}$ & $\mathrm{A}$ & $\mathrm{G}$ & $\mathrm{A}$ & \\
\hline \multicolumn{9}{|l|}{ Gender } \\
\hline Male & 34 & 8 & 28 & 8 & 60 & 0.12 & 0.88 & \multirow{2}{*}{0.297} \\
\hline Female & 30 & 11 & 19 & 11 & 49 & 0.18 & 0.82 & \\
\hline \multicolumn{9}{|c|}{ Age at operation, years } \\
\hline$\leq 40$ & 34 & 9 & 25 & 9 & 59 & 0.13 & 0.87 & \multirow[t]{2}{*}{0.586} \\
\hline$>40$ & 30 & 10 & 20 & 10 & 50 & 0.17 & 0.83 & \\
\hline \multicolumn{9}{|l|}{ Outcome } \\
\hline Primary tumor & 47 & 16 & 31 & 16 & 78 & 0.17 & 0.83 & \multirow[t]{2}{*}{0.105} \\
\hline Recurrence & 17 & 3 & 14 & 3 & 41 & 0.09 & 0.91 & \\
\hline \multicolumn{9}{|l|}{ Histology } \\
\hline Conventional & 61 & 18 & 43 & 18 & 104 & 0.15 & 0.85 & \multirow[t]{3}{*}{-} \\
\hline Chondroid & 2 & 0 & 2 & 2 & 2 & 0 & 1.0 & \\
\hline Dedifferentiated & 1 & 1 & 0 & 0 & 2 & 1.0 & 0 & \\
\hline
\end{tabular}

${ }^{a}$ There was no G/G genotype in neither of the two groups.

to gender, age at operation, whether the tumor was primary or recurrent and tumor histology. As the histology of the majority of the cases was of the conventional type, the associations were not analyzed. There was no association among the other clinical characteristics (Table V).

\section{Discussion}

The present case-control study investigated the Cys1367Arg WRN polymorphism and genotype frequencies in skull base chordoma in a limited number of patients, due to the rarity of this tumor. In the 64 skull base chordomas with rs1346044 SNP, no GG genotype was identified and GA was the minor genotype. These findings were similar to those for the control group of the study by Jiang et al (6) and the results of the PubMed database search. In case of selection bias, the controls of the Jiang et al study were used as a reference, due to their large number (244 controls), well-documented data and identical population investigated (6). The age of 40 years was selected for dividing the chordoma patients in two groups, as it was previously suggested that age may be associated with prognosis in skull base chordomas (2). However, the results did not support the hypothesis that WRN Cys1367Arg SNP is associated with the early development of chordoma. Therefore, there was no positive association between the incidence of skull base chordoma and Cys1367Arg WRN.

The WRN gene is crucial for DNA repair and genomic maintenance (4). The G allele of the rs1346066 of WRN, with the expression of Arg variant of the WRN protein, was reported to enhance the strength of translocation of WRN from the cytoplasm to the nucleus, thus enhancing DNA repair capacity (6). The expression of the WRN gene was reportedly induced in female individuals as compared to males, possibly due to the higher levels of estrogens (8); thus, estrogens may play a protective role against tumor development and aging. However, the precise function of WRN Cys1367Arg SNP has not been fully elucidated and its role remains subject to debate.

Previous studies on aging-related diseases support the protective role of Cys1367Arg. In the Japanese population, Arg1367 has been associated with lower risk of myocardial infarction (9), 
type 2 diabetes mellitus (10) and bone and soft tissue sarcomas (7). In the Han Chinese population, Jiang et al (6) reported that Arg1367 exerts a protective effect against age-related cataract. In a US study, Arg1367 was found to be associated with a decreased risk of non-Hodgkin lymphoma (11). However, other studies reported conflicting results. In a Southeastern Brazilian population, WRN Cys1367Arg was not found to be associated with the risk and prognosis of gliomas (12); in an Israeli population, rs1346044 SNP was not associated with senile cataract (5); and in a German population, the polymorphism of this site was not associated with colorectal cancer (13). However, a protective effect of Cys1367 was reported against osteoporosis in postmenopausal Japanese women (14). Athough most studies support the protective role of Arg1367, there is no consensus among different ethnicities and diseases. Our series showed that the $\mathrm{G}$ and the A alleles were similar between the case and control groups (both in the present study and by Jiang et al) and no association between the SNP rs1346044 and the skull base chordoma was found, which means no such protective role of the 1367Arg was found in the skull base chordoma, which is different from the findings of Nakayama et al (7), although the study population and diseases were similar. However, Nakayama et al studied sarcoma, including a range of bone and soft tissue sarcomas, which is different oncology to the skull base chordoma. Also, this discrepancy may be due to the number of enrolled cases. Our study enrolled only 65 cases, whereas the study by Nakayama et al enrolled 544 cases (7).

The present study exhibited a false positive association between male skull base chordoma patients and Cys1367Arg SNP. However, this conclusion may be affected by selection bias, due to the limited numbers of cases and controls. The effect of gender and hormones on the WRN protein were not definitive; therefore, further studies are required to elucidate the function of the WRN protein and whether it is affected by hormones.

The incidence of chordoma increases with age; $~ 80 \%$ of the patients diagnosed in England between 1979 and 2007 were aged $>50$ years (15). In a meta-analysis including a total of 807 skull base chordomas, the mean age at diagnosis was 51.2 years (16). The mean age of the target population in the present study was similar to those reported by Wu et al (2) (35.6 years) and Di Maio et al (17) (43.6 years), which may be due to the selection bias of single-center analysis. The present study divided the results into 2 groups by a cut-off of 40 years and came up with negative results. Future multi-center investigations are required to resolve this issue.

Although the recurrence of skull base chordoma is largely caused by residual tumor after the first surgery, there is a consensus that the mechanism underlying tumor recurrence may lie with the biological characteristics of the skull base chordomas (18). The WRN gene is a well-conserved gene with an important role in gene protection and has been associated with several tumors (4). The role of Cys1367Arg SNP was also previously investigated $(7,11)$. The present study demonstrated that rs1346033 genotype and allele frequencies did not differ significantly between primary and recurrent skull base chordomas. However, the study design was limited to patient blood samples; tumor samples should be investigated in the future. Due to the relatively short postoperative period ( $\leq 3$ years), no follow-up details were analyzed. Further studies should focus on the association between the Cys1367Arg SNP and the prognosis of skull base chordomas.

In conclusion, the present case-control study did not identify an association between skull base chordoma and WRN Cys1367Arg, at least in the Han Chinese population.

\section{Acknowledgements}

We would like to thank the study subjects for their participation and the staff in Beijing Tian Tan Hospital and Beijing Neurosurgery Institute who contributed to this study.

\section{References}

1. McMaster ML, Goldstein AM, Bromley CM, Ishibe $\mathrm{N}$ and Parry DM: Chordoma: incidence and survival patterns in the United States, 1973-1995. Cancer Causes Control 12: 1-11, 2001.

2. Wu Z, Zhang J, Zhang L, Jia G, Tang J, Wang L and Wang Z: Prognostic factors for long-term outcome of patients with surgical resection of skull base chordomas-106 cases review in one institution. Neurosurg Rev 33: 451-456, 2010.

3. Lee J, Bhatia NN, Hoang BH, Ziogas A and Zell JA: Analysis of prognostic factors for patients with chordoma with use of the California Cancer Registry. J Bone Joint Surg Am 94: 356-363, 2012.

4. Hao S, Feng J, Zhang L, Tang J, Wu Z and Zhang J: Rapid recurrence of petroclival meningioma in Werner syndrome: case report. Clin Neurol Neurosurg 113: 795-797, 2011.

5. Ehrenberg M, Dratviman-Storobinsky O, Avraham-Lubin BR and Goldenberg-Cohen N: Lack of association of the WRN C1367T polymorphism with senile cataract in the Israeli population. Mol Vis 28: 1771-1775, 2010.

6. Jiang S, Hu N, Zhou J, Zhang J, Gao R, Hu J and Guan H: Polymorphisms of the WRN gene and DNA damage of peripheral lymphocytes in age-related cataract in a Han Chinese population. Age (Dordr) 35: 2435-2444, 2013.

7. Nakayama R, Sato Y, Masutani M, et al: Association of a missense single nucleotide polymorphism, Cys1367Arg of the WRN gene, with the risk of bone and soft tissue sarcomas in Japan. Cancer Sci 99: 333-339, 2008.

8. Lee SJ,Lee SH, Ha NC and Park BJ: Estrogen prevents senescence through induction of WRN, Werner syndrome protein. Horm Res Paediatr 74: 33-40, 2010.

9. Ye L, Miki T, Nakura J, et al: Association of a polymorphic variant of the Werner helicase gene with myocardial infarction in a Japanese population. Am J Med Genet 68: 494-498, 1997.

10. Hirai M, Suzuki S, Hinokio Y, et al: WRN gene 1367 Arg allele protects against development of type 2 diabetes mellitus. Diabetes Res Clinic Pract 69: 287-292, 2005.

11. Shen M, Zheng T, Lan Q, et al: Polymorphisms in DNA repair genes and risk of non-Hodgkin lymphoma among women in Connecticut. Hum Genet 119: 659-668, 2006.

12. Pinto GR, Yoshioka FK, Clara CA, et al: WRN Cys1367Arg SNP is not associated with risk and prognosis of gliomas in Southeast Brazil. J Neurooncol 90: 253-258, 2008.

13. Frank B, Hoffmeister M, Klopp N, Illig T, Chang-Claude J and Brenner H: Colorectal cancer and polymorphisms in DNA repair genes WRN, RMI1 and BLM. Carcinogenesis 31: 442-445, 2010.

14. Ogata N, Shiraki M, Hosoi T, Koshizuka Y, Nakamura K and Kawaguchi H: A polymorphic variant at the Werner helicase (WRN) gene is associated with bone density, but not spondylosis, in postmenopausal women. J Bone Miner Metab 19: 296-301, 2001.

15. Whelan J, McTiernan A, Cooper N, Wong YK, Francis M, Vernon S and Strauss SJ: Incidence and survival of malignant bone sarcomas in England 1979-2007. Int J Cancer 131: E508-E517, 2012.

16. Di Maio S, Temkin N, Ramanathan D and Sekhar LN: Current comprehensive management of cranial base chordomas: 10-year meta-analysis of observational studies. J Neurosurg 115: 1094-1105, 2011.

17. Di Maio S, Rostomily R and Sekhar LN: Current surgical outcomes for cranial base chordomas: cohort study of 95 patients. Neurosurgery 70: 1355-1360, 2012.

18. Gagliardi F, Boari N, Riva P and Mortini P: Current therapeutic options and novel molecular markers in skull base chordomas. Neurosurg Rev 35: 1-14, 2012. 